\title{
A modelling approach for the comparison between intensified extraction in small channels and conventional solvent extraction technologies
}

\author{
Davide Bascone, Panagiota Angeli, Eric S. Fraga* \\ Centre for Process Systems Engineering, Department of Chemical Engineering, University \\ College London (UCL), London WC1E 6BT, United Kingdom
}

\begin{abstract}
In this paper, the application of small channels to the extraction separations in spent nuclear fuel reprocessing has been investigated via a modelling approach. The results are compared with conventional liquid-liquid extraction technologies such as mixer- settlers and pulsed columns, using models from the literature.

In the model mass transfer, redox reactions, pressure drop and nuclear criticality are taken into account, as well as manifold and two-phase separator designs for the small-scale technology. The resulting model, posed as an optimisation problem, is a mixed integer nonlinear problem, implemented in the General Algebraic Modeling System (GAMS).

An alternative flowsheet for the codecontamination section of the PUREX process, as a case study, has been investigated. The results show that the smallscale technology could be beneficial, in particular in terms of volumetric mass transfer coefficients, nuclear criticality safety and short residence time, which improves neptunium separation and reduces solvent degradation.
\end{abstract}

Keywords: Liquid-liquid extraction; small channels; spent

nuclear fuel reprocessing; optimisation; process design.

${ }^{*}$ Corresponding author

Preprint submitted to Journal of ${ }^{A} T_{E} X$ Templates

March 24, 2019 


\section{Introduction}

Nuclear power generation could continue to be a viable option for the future, providing lower $\mathrm{CO}_{2}$ emissions than fossil fuels. However, hazardous materials are generated, motivating the need for adequate treatment and disposal. The 5 spent nuclear fuel (SNF) consists of, typically, 96\% uranium and 1\% plutonium [1, although the composition varies with type of fuel, reactor, enrichment, etc. The uranium and plutonium are reusable materials since they may be used as a mixed oxide fuel in a suitable nuclear reactor. The reprocessing of SNF would allow:

- a reduction in the volume of high level waste (HLW);

- a further $25 \%$ of energy from the original uranium [2];

- a reduction in the long-term radiotoxicity of HLW [2].

All the commercial plants use the PUREX process for SNF reprocessing to recover uranium and plutonium by several liquid-liquid extraction operations [3, 4. The PUREX process is generally based on two liquid-liquid extraction cycles: the first one includes codecontamination and $\mathrm{U} / \mathrm{Pu}$ partitioning sections, whilst the second one includes $\mathrm{U}$ and $\mathrm{Pu}$ purification sections. The SNF is first dissolved in a nitric acid solution and then $\mathrm{U}$ and $\mathrm{Pu}$ are recovered by a selective solvent, tri-n-butyl-phosphate (TBP), diluted to 20-30\% (v/v \%) with paraffinic diluent because of its high density and viscosity. The TBP selectivity depends on the oxidation state of the actinide: it is high for the +6 state (U(VI) as $\mathrm{UO}_{2}^{+2}$ ) and the +4 state (e.g. $\mathrm{Pu}(\mathrm{IV})$ as $\left.\mathrm{Pu}^{+4}\right)$, lower for the $5+$ state $(\mathrm{Np}(\mathrm{V})$ as $\mathrm{NpO}^{+2}$ ) and practically zero for +3 and lower states [5]. The chemical equilibria for $\mathrm{U}(\mathrm{VI})$ and $\mathrm{Pu}(\mathrm{IV})$, in the nitrate media, are:

$$
\begin{gathered}
\mathrm{UO}_{2}^{+2}+2 \mathrm{NO}_{3}^{-}+2 \mathrm{TBP} \rightleftharpoons\left[\mathrm{UO}_{2}\left(\mathrm{NO}_{3}\right)_{2}\right] \cdot 2 \mathrm{TBP} \\
\mathrm{Pu}^{+4}+4 \mathrm{NO}_{3}^{-}+2 \mathrm{TBP} \rightleftharpoons\left[\mathrm{Pu}\left(\mathrm{NO}_{3}\right)_{4}\right] \cdot 2 \mathrm{TBP}
\end{gathered}
$$

Besides reactions 1 and 2 extraction of nitric acid in the organic phase inevitably 
occurs [6]:

$$
\begin{gathered}
H^{+}+\mathrm{NO}_{3}^{-}+\mathrm{TBP} \rightleftharpoons\left[\mathrm{HNO}_{3}\right] \cdot \mathrm{TBP} \\
\mathrm{H}^{+}+\mathrm{NO}_{3}^{-}+2 \mathrm{TBP} \rightleftharpoons\left[\mathrm{HNO}_{3}\right] \cdot 2 \mathrm{TBP}
\end{gathered}
$$

In the first section of the modern PUREX processes of La Hague (France) and Rokkasho-mura (Japan) plants, $\mathrm{U}$ and $\mathrm{Pu}$ are typically separated from the other components in the SNF through four main extraction steps [5]:

1. main extraction, where $\mathrm{U}, \mathrm{Pu}$ and low concentrations of other components are extracted from the aqueous feed;

2. Zr and Ru scrub, where $\mathrm{Zr}$ and $\mathrm{Ru}$ are separated from the organic stream loaded with $\mathrm{U}$ and $\mathrm{Pu}$ in the previous step, using diluted nitric acid solution, and the aqueous outlet is recycled to the previous step to increase $\mathrm{U}$ and $\mathrm{Pu}$ recovery;

3. Tc scrub, as the above scrub but removing Tc, through concentrated nitric acid solution;

4. complementary extraction, where $\mathrm{U}$ and $\mathrm{Pu}$ are back extracted from the aqueous phase outoing the previous step and recycled to the main extraction step, to increase $\mathrm{U}$ and $\mathrm{Pu}$ recovery.

This first section of the process, which is the highly active one, is called the "codecontamination" section. It is one of the most challenging sections because of the presence of minor actinides and fission products. Therefore, so particular attention to nuclear criticality safety is needed. Typically, safety is achieved through extraction equipment and with neutron absorbers. An example is pulsed columns, currently used in all commercial plants for this section. Mixer-settlers, widely employed for SNF reprocessing, are not safe by geometry and hence they may be used only for diluted concentrations or low liquid holdup. Difficulties arise from the high extraction and decontamination requirements for $\mathrm{U}$ and $\mathrm{Pu}$. These targets require several recycle streams and different scrubbing operations to remove components that show different thermodynamic behaviour, such as Zr and Tc, as already mentioned. Finally, in the codecontamination area, significant solvent degradation occurs, due to radiolysis, therefore 
reduction of unit operations or volumes, to decrease the contact time and solvent exposure, would be beneficial.

In addition, there are several drawbacks due to the conventional solvent extraction equipment. Pulsed columns and mixer settlers require respectively large amounts of headroom and floor space. Centrifugal extractors are the 45 least reliable equipment from amongst the traditional ones [7. They have low tolerance to solids and require periodic maintenance for the replacement of the rotor and/or the motor. For these reasons their use has been limited [8].

Some of these disadvantages may be overcome if intensified extraction in small-scale contactors is used. The concept of intensification is to decrease size and cost while improving the performance of a process unit. This can be the case of liquid-liquid extractions in small scale contactors where mass transfer is enhanced [9, 10. Other benefits are improved control of hydrodynamics and the reduction in the amounts of hazardous materials involved, which may be particularly advantageous in the nuclear industry.

The use of small channels for liquid-liquid extraction of U(IV) in nitric acid solution, has been investigated in the literature [11, 12, 13, with diameter between 0.5 and $2 \mathrm{~mm}$. Capillaries with Bond numbers lower than 3.37 may be defined as small channels [14, 15]. It is fundamental to properly design a flow distribution network to increase the number of channels ("number up") and 60 therefore increase the throughput. In this work, a 4-level comb-like network will be investigated, as it seems the most suitable manifold type to arrange a large number of channels in a compact stack. Alternative manifolds may be the monolithic reactor type or the tree-like network. Nowadays, the field of flow distribution networks is extensively investigated [16, 17, 18, 19, 20, 21.

${ }_{65}$ Several types of two-phase separators (coalescers, sidestream needles, etc.) are currently being studied, as well. In this work, the design of manifolds, separators and small channels will be optimised. The resulting size of these elements provide indications on possible future investigations.

An alternative process flow diagram for the codecontamination section of the PUREX process is presented below and optimised on the basis of economic 
criteria. The short-cut models used, described in the following section, allow for a preliminary process design and optimisation. These type of models, widely used in Process Engineering, are not as computational expensive as CFD models, although with the latter a more detailed analysis of a single unit operation

75 is possible. Applications of small-scale contactors, pulsed columns and mixersettlers are investigated, using mathematical models developed in the literature. The performance and the main chemical engineering attributes of the liquidliquid extraction technologies are investigated and compared.

\section{Summary of mathematical models}

Mathematical models developed in the literature have been used to design, optimise and compare the liquid-liquid extraction processes. In particular, technologies investigated are the novel small-scale process units, pulsed columns and mixer-settlers. In this section, the main aspects of the models used are summarised. Assumptions considered to develop the mass balance model, as well

85 as correlations used to predict thermodynamics, mass transfer, hydrodynamics, pressure drops, flow network design, redox reactions and criticality, are stated.

\subsection{Chemistry}

The system, suitable for SNF reprocessing, involves the following components: $\mathrm{U}(\mathrm{VI}), \mathrm{Pu}(\mathrm{IV}), \mathrm{Zr}, \mathrm{Ru}, \mathrm{Tc}, \mathrm{Np}(\mathrm{IV}), \mathrm{Np}(\mathrm{V})$ and $\mathrm{Np}(\mathrm{VI}), \mathrm{HNO}_{3}$. Nitrous

acid $\mathrm{HNO}_{2}$ has also been included, to simulate the radiolytic degradation of $\mathrm{HNO}_{3}$. The distribution coefficients of all components have been estimated according to the sources listed in Table 1.

A difficult task in the PUREX process is the control of Np since Np exists in nitric acid solution with three different, easily inter-convertible, oxidation states, $\mathrm{Np}(\mathrm{IV}), \mathrm{Np}(\mathrm{V})$ and $\mathrm{Np}(\mathrm{VI})$ :

$$
\begin{gathered}
\mathrm{NpO}_{2}^{+}+0.5 \mathrm{NO}_{3}^{-}+1.5 \mathrm{H}^{+} \rightleftharpoons \mathrm{NpO}_{2}^{2+}+0.5 \mathrm{HNO}_{2}+0.5 \mathrm{H}_{2} \mathrm{O} \\
2 \mathrm{NpO}_{2}^{+}+4 \mathrm{H}^{+} \rightleftharpoons \mathrm{Np}^{4+}+\mathrm{NpO}_{2}^{2+}+2 \mathrm{H}_{2} \mathrm{O}
\end{gathered}
$$


Reaction rates are taken from the literature [22, 23, 24, 25]. Details of the equations implemented to calculate all distribution coefficients and reaction rates are given by Bascone et al. 26].

Table 1: Sources for the calculation of distribution coefficients used in this work.

\begin{tabular}{ll}
\hline Component & Source \\
\hline $\mathrm{U}(\mathrm{VI}), \mathrm{Pu}(\mathrm{IV}), \mathrm{HNO}_{3}$ & Richardson and Swanson[6] \\
$\mathrm{Zr}, \mathrm{Ru}$ & Natarajan et al. $[27]$ \\
$\mathrm{Tc}$ & Asakura et al. $[28]$ \\
$\mathrm{Np}(\mathrm{IV}), \mathrm{Np}(\mathrm{VI})$ & Benedict et al. [29], coefficients re-calculated by $\mathrm{Ku}-$ \\
& mar et al. $[30]:$ \\
$\mathrm{Np}(\mathrm{V})$ & Nominal value of $0.01[23]:$ \\
$\mathrm{HNO}_{2}$ & Uchiyama et al. $[31]$ \\
\hline
\end{tabular}

\subsection{Nuclear criticality safety}

SNF contains fissile materials. Hence, nuclear criticality safety must be guaranteed in the reprocessing. The nuclear criticality safety should be rigorously analysed by codes that solve the time-dependent transport equation of the neutron flux. However, these types of codes are too computational expensive and not suitable for flowsheet optimisation. Here, the migration-area approximation has been used to estimate the effective multiplication factor $k_{\text {eff }}$, i.e. the ratio between the number of neutrons produced by fission in one neutron generation and the number of neutrons lost in the previous neutron generation, due to absorption and leakage [32. Hence, by definition, to guarantee subcriticality, the $k_{\text {eff }}$ must be lower than 0.95 , a value lower than 1 , which indicates criticality, for safety reasons. This method is not sufficient to determine nuclear criticality safety, but it can provide a first measure and valuable insight to compare liquid-liquid extraction technologies. 

with diameter varying from $0.5 \mathrm{~mm}$ to $1 \mathrm{~mm}$, the slug flow is stable in polytetrafluoroethylene (PTFE) capillaries for aqueous to organic flow rate ratios from $0.17-0.33$ to $2.5-5$. The maximum reported superficial velocity of the mixture, for slug flow, is approximately $4 \mathrm{~cm} \mathrm{~s}^{-1}$. The flow behaviour of the system 135 fully characterised. Hence, the most conservative limits for aqueous to organic

where $\sum R_{i, k} \varphi$ is the sum of all chemical reactions involving the component $i$ in the phase $k$. Eq. 7 is converted to a set of algebraic equations through the application of a fourth order Runge-Kutta method. Eq. 7 does not take into account the effect of the flow rate ratio, therefore an empirical equation has been used to consider the latter [26].

The volumetric mass transfer coefficient $k_{L} a$ has been calculated as suggested by Kashid et al. [33]:

$$
k_{L} a=0.88 \frac{v_{m i x}}{L} C a^{-0.09} R_{m i x}^{-0.09}\left(\frac{D}{L}\right)^{-0.1}
$$

where $v_{m i x}$ is the superficial velocity of the two-phase flow, $D$ and $L$ are respectively the diameter and length of the channels. The equation above showed good agreement with experimental data of uranium extraction in small channels up to $2 \mathrm{~mm}$ using a mixture of ionic liquid with TBP, although with recalculated parameters [13. In this work, as the physical system is similar to the one used by Kashid et al., the original parameters have been used.

Eq. 8 is valid for slug flow. According to Kashid and Agar 34, for the system water-cyclohexane with both main capillary and Y-junction channels TBP-kerosene/nitic acil solution, in small channels up to 2 min, has not been 
flow ratios (0.33-2.5) and velocities reported by Kashid and Agar [34 have been used as bounds for these variables in the model.

Channels are assumed to be hydrophobic to disperse the aqueous phase, as in the extraction channels used for the main extraction to protect the solvent from contaminants (all the solids are directed with the dispersed phase) [5]. The cost of channels and manifolds, assumed as first estimation equal to the sum of channels and mixing tees, is estimated using prices provided by CM Scientific Laboratory Supplies [35] for transparent polymers. These costs are multiplied by a material factor $(0.1$, based on the current market for nontransparent polymers such as PTFE), to reasonably compare costs between liquid-liquid extraction technologies. A linear scaling factor is assumed [36]. Operating cost consists of pumping cost. See Bascone et al. 26] for the full set of equations..

\subsubsection{Unit description}

The flow network design is essential to distribute the organic and aqueous phases in the parallel small channels ensuring a good flow uniformity. Here, the methodology developed by Commenge et al. 21] has been used to design the manifolds. This methodology, based on the electrical resistances network model and assuming isothermal and laminar flow, allows to design a comb-like manifold, which seems the most appropriate manifold to arrange a large number of small channels in a compact stack [21]. Due to the throughput investigated in this work, a four-level structure has been considered (see Figure 1). The $4^{\text {th }}$ level is the single largest distributor/collector, which connects the different modules. The $3^{\text {rd }}$ level distributes/collects the single phase flow through the stack of plates, the $2^{\text {nd }}$ one distributes/collects the single phase flow through the channels of the plates. The $1^{\text {st }}$ level consists of the small channel extractors and it is shared by the two manifolds (one for each phase) through a mixing junction. At the end of level 1, a sidestream channel is used to separate the dispersed plug from the continuous slug, as described by Scheiff et al. [37. Each channel or distributor in each level is defined as "element".

A multi-stage counter-current configuration has been considered, to achieve 


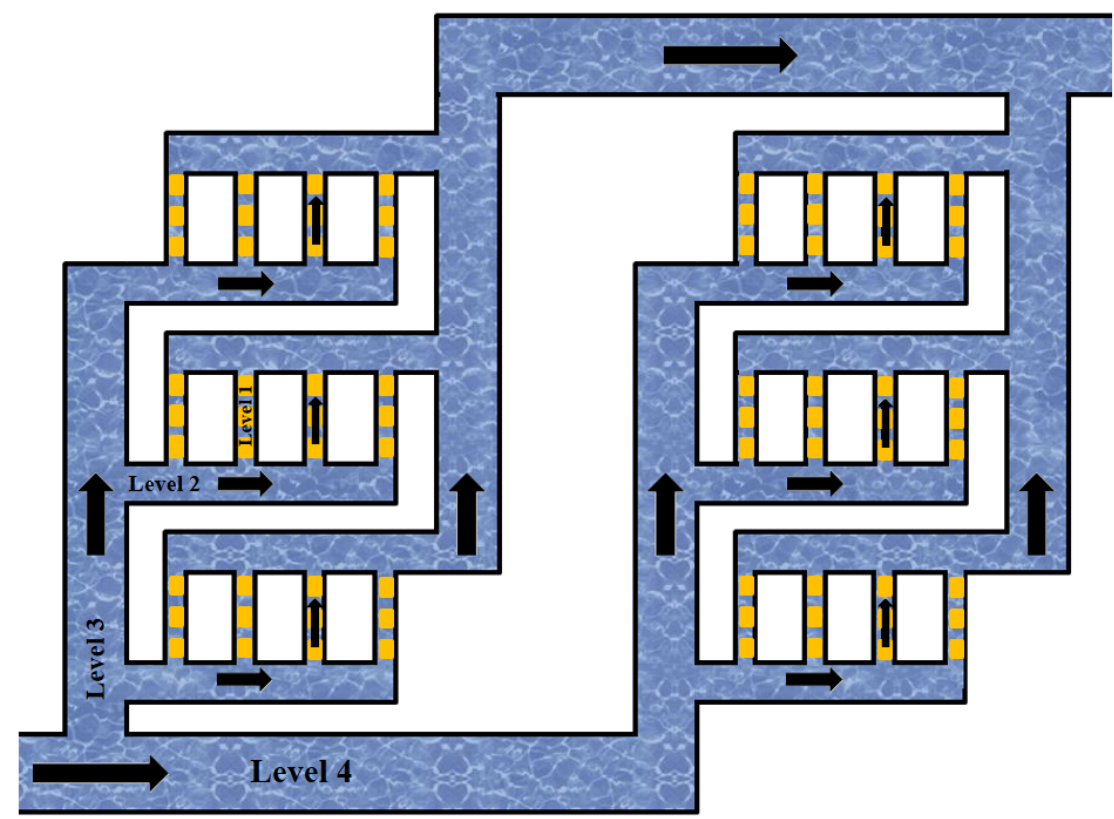

Figure 1: Schematic of the four-level network. In this sketch, one element in the level 4, two in the level 3, three in the level 2 and four in the level 1 are shown. The level 1 consists of the small extractors (yellow dispersed plugs are shown).

a high extraction efficiency. Two manifolds are required for each stage, one for each phase. For more details, see [26].

\subsection{Modelling of mixer-settler units}

Mixer-settlers have been widely investigated in the last 60 years. Assuming homogeneous concentrations and steady state, the mass balance model for the component $i$ in the phase $k$ is given by:

$$
\begin{aligned}
& \text { mixer } \quad \dot{V}_{k}\left(C_{i, k}^{i n}-C_{i, k}\right)+k_{L} a V\left(C_{i, k}-C_{i, k}^{e q}\right)+\sum R_{i, k} \varphi_{k} V_{\text {mix }}=0 \\
& \text { settler } \quad \dot{V}_{k}\left(C_{i, k}^{i n}-C_{i, k}\right)+\sum R_{i, k} \varphi_{k} V_{\text {set }}=0
\end{aligned}
$$


Mixer-settlers are modelled considering a counter-current configuration, ac-

at the bottom, stage 1

$$
\left(1+\alpha_{a q}\right)\left(C_{2, a q}^{i}-C_{1, a q}^{i}\right)-\frac{N_{o c}}{N}\left(C_{1, a q}^{i}-C_{1, a q}^{i, e q}\right)+\sum R_{a q}^{i} \varphi_{a q} V=0
$$

The Sauter mean diameter has been calculated by the Calderbank equation for a stirred vessel with a four bladed-impeller [41].

The operating cost is the agitation cost. The maximum settling velocity of dispersed drops, required to design the settlers, is assumed equal to $1.5 \mathrm{~mm} \mathrm{~s}^{-1}$ 42. The capital costs of mixer-settler units are estimated considering the costs of vessels and agitators, as suggested by Seider et al. 43 .

\subsection{Modelling of pulsed column units}

A stagewise mass balance model is used for pulsed columns, as suggested by Steiner [4], because the distribution coefficients are not constant. Steady state conditions have been assumed. Hence, the mass balance for component $i$ in the aqueous phase is given by 
typical stage $n$

$$
\begin{aligned}
& \left(1+\alpha_{a q}\right)\left(C_{n+1, a q}^{i}-C_{n, a q}^{i}\right)+\alpha_{a q}\left(C_{n-1, a q}^{i}-C_{n, a q}^{i}\right)-\frac{N_{o c}}{N}\left(C_{n, a q}^{i}-C_{n, a q}^{i, e q}\right)+ \\
& +\sum R_{a q}^{i} \varphi_{a q} V=0
\end{aligned}
$$

at the top, stage $N$

$$
\begin{aligned}
& C_{i n, a q}^{i}+\alpha_{a q} C_{N-1, a q}^{i}-\left(1+\alpha_{a q}\right) C_{N, a q}^{i}-\frac{N_{o c}}{N}\left(C_{N, a q}^{i}-C_{N, a q}^{i, e q}\right)+ \\
& +\sum R_{a q}^{i} \varphi_{a q} V=0
\end{aligned}
$$

$N_{o c}$ is the number of transfer units (which is affected by the column height, as $\left.N_{o c}=k_{L} a H / v\right), \alpha$ is equal to $N / P e-0.5$, where $P e$ is the Peclet number calculated as $v \Delta H / E, \Delta H$ is the plate spacing, assumed equal to $5 \mathrm{~cm}$ (typical value [45, 46]), $E$ is the axial dispersion coefficient. Hence, $\alpha$ represents the deviation from plug flow due to back-mixing. In each stage, perfect mixing is assumed. The mass balance for component $i$ in the organic phase is calculated similarly [44].

Expressions for the calculation of mass transfer coefficients of U(VI), $\mathrm{Pu}(\mathrm{IV})$ and $\mathrm{HNO}_{3}$, as well as hydrodynamics correlations, are taken from [45, 47]. The mass transfer coefficient for $\mathrm{U}(\mathrm{VI})$ during extraction (from dispersed aqueous phase to continuous organic phase) is calculated by the following correlation:

$$
k_{L}=2.8 \times 10^{7}(1 / P e)^{1.8}+\frac{3.55 \times 1014}{m}(1 / P e)^{2.95}
$$

In Eq. 15 the Peclet number is calculated as $v_{t} d_{p} / \mathscr{D}$ [47. Similar expressions have been used for mass transfer of $\mathrm{Pu}(\mathrm{III}), \mathrm{U}(\mathrm{IV}), \mathrm{Pu}(\mathrm{IV})$ and $\mathrm{HNO}_{3}$.

Conditions of insufficient pulsation and flooding are calculated as suggested by Berger and Walter [48. Ranges for pulse frequency and amplitude are taken from Kumar and Hartland [46. To estimate the operation cost, pumping cost and power requirement for pulse generations are considered [49. Capital costs are calculated as suggested by Seider et al. for columns (considering vessels, plates and platforms) 43 . Costs of the top and bottom settlers are also included. The cost of other equipment and instrumentation needed, such as the 
neutron absorber and the pulsator, has not been included due to the lack of information for confidentiality reasons.

\section{Case study}

A case study is now presented. The goal is to investigate the potential benefits and drawbacks that the small-scale contactor could bring to SNF reprocessing when compared against traditional liquid-liquid extraction equipment. The process investigated is an alternative flowsheet for the modern codecontamination section (described in Section 1) where the two scrubbing steps, typically with the first step using a $1.5 \mathrm{M} \mathrm{HNO}_{3}$ solution and the second step a $10 \mathrm{M}$ $\mathrm{HNO}_{3}$ solution, are merged in a single scrubbing step. The possibility of reducing the number of unit operations while achieving the minimum separation required, using a nitric acid solution of intermediate concentration in the single scrubbing step and varying equipment design and operating conditions, is investigated. The process flow diagram is shown in Figure 2. The aqueous stream leaving the scrubbing step could be either recycled to the main extraction (step 1 in Figure 22, fed to the complementary extraction (step 3) or split between these two steps. The design variable $\chi$, which can vary between 0 and 1 , describes the fraction of aqueous stream recycled to step 1 and can be: $\chi \dot{V}_{a q}$ is fed to step $1,(1-\chi) \dot{V}_{a q}$ to step 3 . The organic stream from step 3 is entirely recycled to step 1, which is the standard approach in the modern PUREX processes [5. In the commercial plants there are also unit operations to recover the small amount of TBP ended up in the aqueous phase. However, as the mass transfer of TBP is neglected in this work (very small solubility in nitric acid solution), these unit operations are not included in this case study.

The aqueous stream leaving step 1 is categorised as HLW. These radioactive effluents typically undergo vitrification or solidification for final storage and, then, disposal. In the aqueous stream leaving step 3 the concentration of fissile materials is significantly lower compared to the aqueous stream leaving step 1 . Hence, the handling of the aqueous stream leaving step 3 is not more complex 
than the typical handling and disposal of the one leaving step 1.

\subsection{Optimisation framework}

The goal is to optimise the process shown in Figure 2 minimising the total annualised cost $(T A C)$, which includes operating expenditure $(O p E x)$ and annualised capital expenditure (ACapEx) :

$$
T A C=O p E x+A C a p E x
$$

A payout time of 5 years has been assumed (typical values between 5 and 10 years [50]) with a $5 \%$ interest rate. Free variables are all the design variables related to the geometry of the equipment. For the small-scale contactor, these variables are the length and width or diameter (for small extractors and distribution channels) of each level of the flow network and the number of stages and elements within each level (integer variables). For mixer-settlers, the design variables are width, depth and length of mixers and settlers and the diameter of the impeller and number of stages. For pulsed columns, the design variables are column diameter and the number of perforated plates (integer variable), which will determine the column height. Further free variables are the operating conditions: the flow rate of all inlet streams except the feed, both aqueous and organic, as well as nitric acid concentration of the aqueous stream entering step 2. Also, the impeller speed is a free variable in the mixer-settler model and the pulse frequency and amplitude are free variables in the pulsed column. Constraints such as minimal requirements for recovery $(R e c)$ of $\mathrm{U}$ and $\mathrm{Pu}$, or decontamination factor $(D F)$ of hazardous components such as $\mathrm{Zr}$ and $\mathrm{Ru}$, are included in the optimisation problem. The recovery is defined as the amount of metal in the product stream divided by the amount of metal in the feed, whilst the decontamination factor is the ratio between the amount of a certain contaminant in the feed and in the product stream. All the inequality constraints included in the optimisation problem are shown in Table 2 .

The optimisation problem has been solved three times, one for each technology, to identify their potential advantages and disadvantages. Inputs of the 

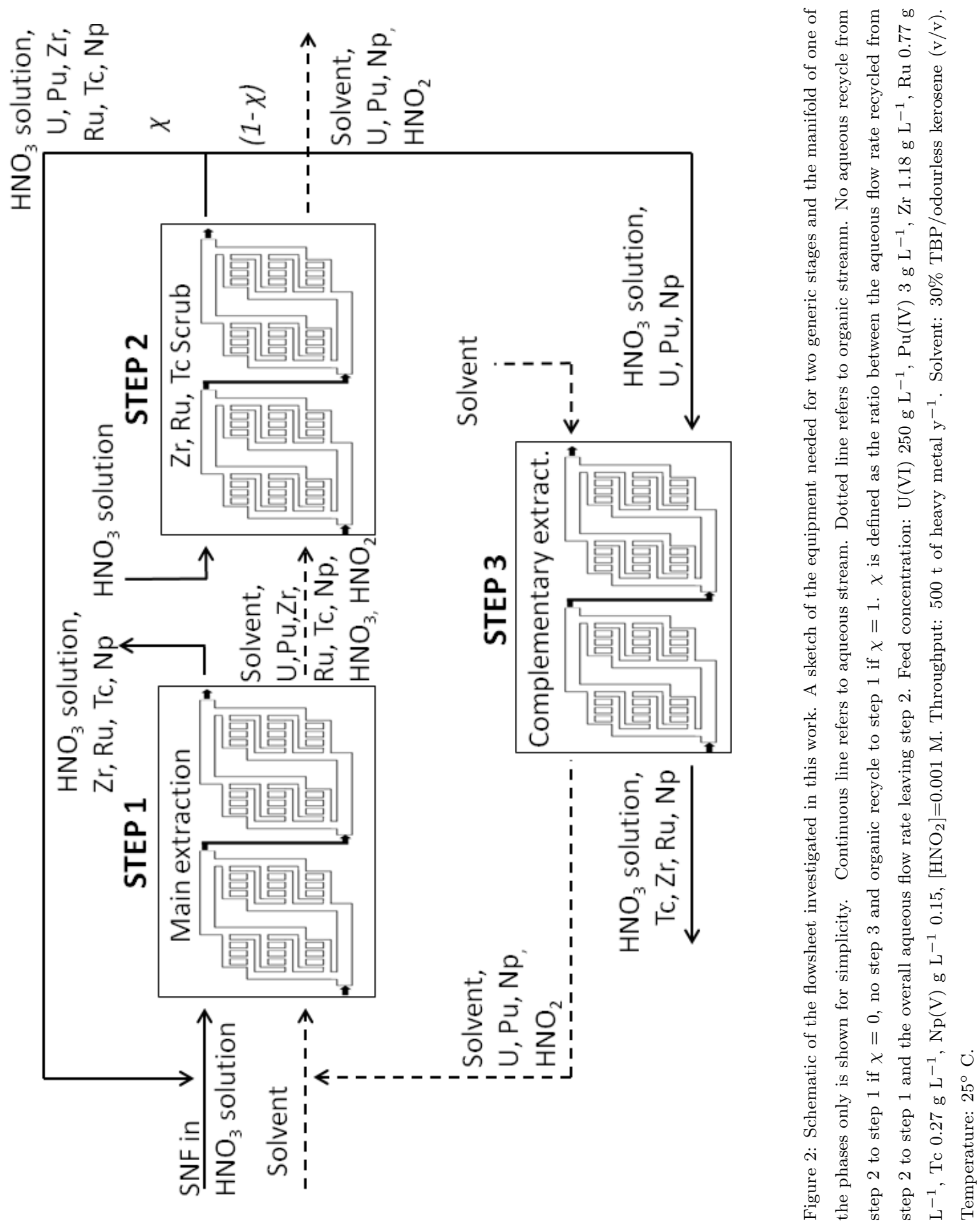
Table 2: Inequality constraints used.

\begin{tabular}{ll}
\hline Inequality constraints & Comment \\
\hline$R e c_{U} \geq 0.99$ & Minimum uranium recovery \\
$R e c_{P u} \geq 0.99$ & Minimum plutonium recovery \\
{$[U]_{\text {aq, out }}^{\text {Step }} \leq 1.68 \mathrm{M}$} & Maximum typical uranium concentration in \\
& the HLW from step 1 \\
$D F_{Z r} \geq 10^{4}$ & Typical requirement [5] \\
$D F_{R u} \geq 10^{4}$ & Typical requirement [5] \\
$D F_{T c} \geq 10$ & Typical requirement [5] \\
$k_{e f f} \leq 0.95$ & Nuclear criticality safety \\
\hline
\end{tabular}

problem are throughput, feed concentration, temperature and materials. The same feed concentration and rate, $500 \mathrm{t}$ of heavy metals per year $\left(\mathrm{MTHM}^{-1}\right)$, have been used for all cases. This throughput is an average one for SNF from Light Water Reactors (600 MTHM y ${ }^{-1}$ at THORP in Sellafied, $400 \mathrm{MTHM} \mathrm{y}^{-1}$ at Mayak in Russia [51]). The feed concentration of the dissolved SNF in nitric acid solution is the typical one from a Light Water Reactor.

280 The combination of the nonlinear models and the integer variables leads to the optimisation problem which is described as a mixed integer nonlinear programme. This problem is implemented and solved in GAMS [52]. The integer variables are the number of stages in the models for intensified extraction in small channels and mixer-settlers, whilst in pulsed columns the integer variable represents the number of perforated plates. The optimisation problems defined for each of the three configurations have been solved using the SBB (Simple Branch and Bound) solver. The relative gap criterion (OPTCR) is $1 \%$.

The optimisation model for the small-scale contactor is the largest, with approximately 270000 equations, because of the 50 grid points used to convert 290 the differential mass balance equation 7 to a set of algebraic equations. For the pulsed column alternative, there are approximately 20000 equations due 
to the 50 theoretical stages, which must not be confused with the number of actual compartments (which vary with the height of the column and the number of plates). For the mixer-settler configuration, there are approximately 6000 equations.

\section{Results}

The main results of the optimisation problems are shown in Table 3 These results allow a first comparison between the extraction technologies, which is the aim of this work. A general comparison of the most important chemical engineering attributes of all liquid-liquid extractors, including the centrifugal extractor, are shown in Figure 3. In the latter, ratings for conventional technologies are taken from Law [7, except the last four rows where the ratings are based on the results obtained in this work. The ratings for small channels have been estimated assuming proportionality between the typical values of the attribute for the traditional technologies (provided by Arm et al. [8]), its rating (provided by Law), the value of the attribute of small channels achieved in this work and its rating. If values for conventional technologies are not provided by Arm et al., the values obtained in this work are used. Where the attribute cannot be directly measured, such as ease of scale-up or process flexibility, a first estimation is provided by the authors, referring to the ratings given by Law for the other equipment.

According to the models, the pulsed column height in the main extraction is significantly shorter than the typical value (12 $\mathrm{m} 8$ ]), due to the operating conditions achieved by the optimisation problem. As a result of this design, the pulsed column is the most cost effective technology.

The optimal process design using intensified extraction in small channels is shown in Tables 4 6, whereas the designs of conventional technologies are illustrated in Tables 749 The small contactor is the only one which makes use of the complementary extraction, step 3. The flow diagrams achieved by the optimisations are further discussed in section 4.1. Based on the process 
designs, the most important criteria (i.e. economics, size, safety, mass transfer, $\mathrm{Np}$ control) and further considerations are discussed in sections 4.24 .7

\subsection{Optimal flowsheet}

Using the conventional technologies, the optimal flowsheet does not include step 3, i.e. $\chi=1$. This choice reduces the total annualised cost for the conventional technologies. With the small scale technology, the optimal value of $\chi$ is 0.69 , therefore $31 \%$ of the aqueous stream leaving step 2 undergoes treatment in step 3 . There is an approximately $3 \%$ difference between the case with $\chi=1$ and the optimal solution, i.e $\chi=0.69$. When $\chi$ is 1 and step 3 is not included, the first step involves 5 stages and the second step 3 stages, whilst the optimal solution $(\chi=0.69)$ involves 4 stages in steps 1 and 2 but 1 stage in step 3, as shown in Table 6. Therefore, there is the same total number of stages in both cases for the first two steps, plus a small unit for step 3 (single stage) in the optimal flowsheet, similar overall cost and operating conditions and similar designs. The optimal solution $(\chi=0.69)$ involves slightly lower overall capital cost, mainly manifold cost, as a result of the $30 \%$ more cost effective manifold for step 1 (one stage less than the case with $\chi=1$ ). These differences in the total annualised cost and process design show the benefits of the modular nature and hence the flexibility of the small-scale technology. 


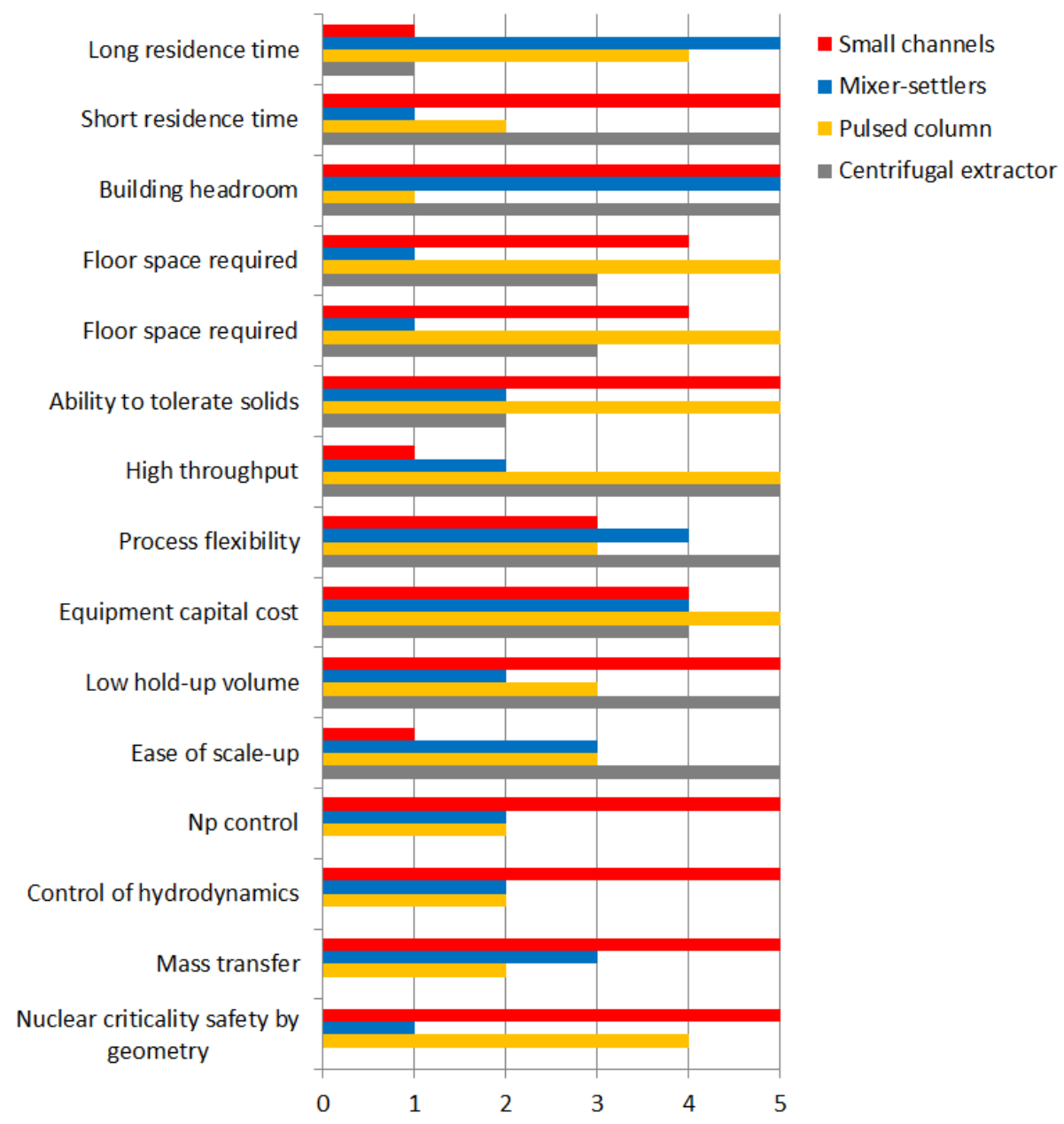

Figure 3: General comparison of small channels, mixer-settler, pulsed columns and centrifugal extractors. Ratings: $5=$ superior, $4=$ good, $3=$ average, $2=$ below average, $1=$ poor. The ratings for the conventional technologies (except the last four rows) are taken from Law 7 . Process flexibility includes factors such as aqueous to organic flow ratio and turndown in flowrate. The long residence time is considered an advantage when it is required by the process kinetics, the short residence time is considered an advantage to limit the solvent degradation [7]. Ratings for Np control, control of hydrodynamics, mass transfer and nuclear criticality safety are not reported for centrifugal extractors, as they have not been investigated or provided by Law [7. 
Table 3: Comparison of small channels, pulsed columns and mixer-settlers. The total residence time is referred to the two-phase flow in the whole process.

\begin{tabular}{lrrr}
\hline Criteria & $\begin{array}{r}\text { Small } \\
\text { channels }\end{array}$ & $\begin{array}{r}\text { Mixer- } \\
\text { settler }\end{array}$ & $\begin{array}{r}\text { Pulsed } \\
\text { colum }\end{array}$ \\
\hline Total annualised cost $\left[£ \mathrm{y}^{-1} \times 10^{3}\right]$ & 39 & 58 & 27 \\
Recycle ratio $\chi$ & 0.69 & 1.00 & 1.00 \\
Total solvent flow rate $\left[\mathrm{L} \mathrm{h}^{-1}\right]$ & 645 & 617 & 940 \\
Total liquid volume $[\mathrm{L}]$ & 82 & 350 & 239 \\
Total residence time $[\mathrm{min}]$ & 0.8 & 18.4 & 6.3 \\
Np decontamination factor & 29.9 & 3.4 & 1.7 \\
Zr decontamination factor & $1.0 \times 10^{4}$ & $1.0 \times 10^{4}$ & $1.3 \times 10^{4}$ \\
Ru decontamination factor & $1.1 \times 10^{6}$ & $3.6 \times 10^{7}$ & $9.0 \times 10^{9}$ \\
Tc decontamination factor & 10.0 & 10.0 & 10.0 \\
$\mathrm{U}$ recovery $(\%)$ & 99.2 & 99.9 & 99.6 \\
Pu recovery $(\%)$ & 99.0 & 99.0 & 99.0 \\
Average $k_{L} a\left[\mathrm{~s}^{-1}\right]$ & 0.35 & 0.19 & 0.11 \\
Criticality safety: $k_{\text {eff }}$ & 0.3 & 0.7 & 0.6 \\
\hline
\end{tabular}


Pulsed columns were expected to be the least expensive conventional equipment [7]. The low cost of pulsed columns achieved in this work is due to the short height achieved, significantly shorter than the typical height reported in the literature for SNF reprocessing [8]. This is the result of the dilution of the feed with the aqueous stream leaving step 2 and the increased solvent flow rate (see Table 3), which improves extraction at the expense of a high usage of solvent and the related costs. These operating conditions lead to height and volume lower than expected. As the costs of some equipment has not been included in the calculation, the overall cost of pulsed columns may be significantly underestimated.

The operating cost of small channel contactors, the pumping cost, is negligible if compared to the annualised capital cost. The proper design of the manifolds reduces the pressure drop along distributors and collectors, minimising the pumping power required. In all steps, approximately $94 \%$ of the overall pressure drop in the small scale technology occurs in the mixing junction at the entry of the small extractor ( $1^{\text {st }}$ level of the manifold), where the two phases join. This result confirms the very small pressure drop achieved with laminar flow in the distributors and collectors. Compared to the operating costs required by conventional technologies, the resulting operating cost for small channels is one order of magnitude lower.

\subsection{Size}

The size of equipment has a direct impact on safety as the amount of hazardous liquids present depends on the equipment volume. Furthermore, for equal flow rates, smaller sizes lead to shorter residence times. The residence time affects the solvent degradation and hence the solvent regeneration and makeup costs. The optimal design achieved with small channels involves the lowest total liquid volume among all the alternatives considered, about one third of the one required by the pulsed column. 
Compared with the volume of mixer-settlers typically used in the nuclear

\subsection{Safety}

In terms of nuclear criticality safety, the results confirm that the small scale technology is safer than mixer-settlers and pulsed columns by geometry. Therefore, the use of small channels in the highly active section, in place of pulsed columns, can be a viable option for future SNF reprocessing. The low effective multiplication factor $k_{\text {eff }}$ is due to the high surface area to volume ratio and 
the small liquid holdup in each small channel. The value of $k_{\text {eff }}$ in small chan-

\subsection{Np control}

The control and separation of neptunium is improved using small channels because the residence times are short; this is a result of the efficient mass trans- 
fer. The short residence time is not sufficient to allow high conversion of $\mathrm{Np}(\mathrm{V})$,

unextractable, to $\mathrm{Np}(\mathrm{VI})$, easily extractable. Formation of $\mathrm{Np}(\mathrm{IV})$, with these nitric acid concentrations, is negligible as expected [55. The decontamination factor of $\mathrm{Np}$, i.e. the ratio between the amount of $\mathrm{Np}$ in the feed and the one in the product stream (the organic stream outoing step 2) is around 30, which means that over $95 \%$ is removed with the high level wastes. Using conventional technologies, the decontamination factor for $\mathrm{Np}$ is between 1.7 (pulsed columns) and 3.4 (mixer-settlers), i.e. Np removal respectively of $40 \%$ and $70 \%$. The presence of Np complicates the next cycles of the PUREX process (not investigated in this case study), which could hence benefit from the employment of small channels.

\subsection{Other results}

One of the most important advantages of the small-scale technology is the low solvent degradation due to the short residence time of the two-phase flow in the contactor, approximately 5 seconds for each stage of each step. Consequently, the need of solvent makeup and the solvent regeneration costs are reduced. Solvent regeneration requires expensive evaporation and rectification operations [5].

Criticality, process volumes, costs, solvent exposure and the other aspects discussed above are not the only factors to consider for the application of liquidliquid extraction equipment in the nuclear industry. Process flexibility could benefit from using small channels: turndown in flow rate and change in the aqueous to organic flow ratio could be achieved by decreasing the number of parallel channels although the consequent possibility of flow maldistribution along the channels (i.e. non-uniform flow distribution in the channels) would need to be considered [56. Small channels could be used in any section of the process, being safe from nuclear criticality by geometry.

To increase throughput, scale up of the small scale contactors by increasing the sizes of the channels can be considered. Above a certain size, however, the mass transfer coefficient could decrease and the resulting extraction will be less 
efficient. Scale-out, or numbering-up, is the use of more channels in parallel to achieve higher throughput. In scale-out, channel sizes are not modified. However, scale-out is limited by the design of the manifold: a very large and not practical number of channels may be required and high throughput could be difficult to achieve. Using small channels, scale-up and scale-out must be combined to increase throughput and ensure high separation performance. The maximum processing capacity depends on the type of manifold used. With the 4-level comb-like network used here and throughput of 500 tons of heavy metals per year, the largest step involves about 4650 parallel channels. Assuming a linear relationship between feed and number of channels needed, which is reasonable for this technology, with an increase of the feed by 100\%, approximately 10000 channels may be required. However, these numbers of channels regard only the first section of the PUREX process, which is the one investigated here. The following process sections may require unpractically large numbers of channels. Hence, to properly identify the maximum throughput using small channels, the entire process should be investigated.

Contrary to technologies with moving parts, such as mixer-settlers and centrifugal extractors, small channels could tolerate solids. With diameters of the channels in the order of millimetres, the risk of occlusion is minimised. 
Table 4: Optimal design of flow network for intensified extraction in small channels. Steps: 1 $=$ main extraction, $2=$ scrubbing, $3=$ complementary extraction.

\begin{tabular}{|c|c|c|c|c|c|}
\hline Step & Phase & Scale & No. elements & $\begin{array}{r}\text { Length } \\
{[\mathrm{cm}]}\end{array}$ & $\begin{array}{r}\text { Diameter/ } \\
\text { Width }[\mathrm{cm}]\end{array}$ \\
\hline 1 & aq.,org. & 1 & 47 & 10.0 & 0.2 \\
\hline 1 & aq. & 2 & 11 & 27.6 & 0.7 \\
\hline 1 & aq. & 3 & 9 & 19.6 & 1.1 \\
\hline 1 & aq. & 4 & 1 & 26.9 & 6.1 \\
\hline 1 & org. & 2 & 11 & 27.6 & 0.8 \\
\hline 1 & org. & 3 & 9 & 23.2 & 1.5 \\
\hline 1 & org. & 4 & 1 & 35.1 & 4.1 \\
\hline 2 & aq.,org. & 1 & 40 & 10.0 & 0.2 \\
\hline 2 & aq. & 2 & 11 & 23.4 & 0.6 \\
\hline 2 & aq. & 3 & 9 & 17.8 & 1.0 \\
\hline 2 & aq. & 4 & 1 & 23.0 & 4.0 \\
\hline 2 & org. & 2 & 11 & 23.4 & 0.7 \\
\hline 2 & org. & 3 & 9 & 20.7 & 1.4 \\
\hline 2 & org. & 4 & 1 & 33.9 & 4.1 \\
\hline 3 & aq.,org. & 1 & 45 & 10.0 & 0.2 \\
\hline 3 & aq. & 2 & 8 & 26.4 & 0.6 \\
\hline 3 & aq. & 3 & 3 & 13.3 & 1.0 \\
\hline 3 & aq. & 4 & 1 & 5.7 & 1.4 \\
\hline 3 & org. & 2 & 8 & 26.4 & 0.7 \\
\hline 3 & org. & 3 & 3 & 15.4 & 1.3 \\
\hline 3 & org. & 4 & 1 & 7.9 & 1.9 \\
\hline
\end{tabular}


Table 5: Optimal design of phase separator for intensified extraction in small channels. Steps: $1=$ main extraction, $2=$ scrubbing, $3=$ complementary extraction.

\begin{tabular}{rrrrr}
\hline Step & Stream & $\begin{array}{r}\text { Length } \\
{[\mathbf{c m}]}\end{array}$ & $\begin{array}{r}\text { Diameter } \\
{[\mathbf{c m}]}\end{array}$ & Material \\
\hline 1 & Mainstream & 2.9 & 0.2 & PTFE \\
1 & Sidestream & 1.0 & 0.1 & Stainless steel \\
2 & Mainstream & 2.6 & 0.2 & PTFE \\
2 & Sidestream & 1.0 & 0.1 & Stainless steel \\
3 & Mainstream & 2.8 & 0.2 & PTFE \\
3 & Sidestream & 1.0 & 0.1 & Stainless steel \\
\hline
\end{tabular}

Table 6: Optimal design of liquid-liquid extraction using small channels. Steps: $1=$ main extraction, 2 = scrubbing, $3=$ complementary extraction.

\begin{tabular}{lrrr}
\hline Variable & Step 1 & Step 2 & Step 3 \\
\hline No. stages & 4 & 4 & 1 \\
Aq. to org. flow ratio & 0.63 & 0.42 & 0.51 \\
{$\left[\mathrm{HNO}_{3}\right]$ aqueous scrubbing } & N.A. & 4.7 & N.A. \\
stream [M] & & & \\
\hline
\end{tabular}

Table 7: Optimal geometry of mixer-settlers. Steps: $1=$ main extraction, $2=$ scrubbing.

\begin{tabular}{lrrrrr}
\hline Step & Unit & $\begin{array}{r}\text { Width } \\
{[\mathbf{m}]}\end{array}$ & $\begin{array}{r}\text { Length } \\
{[\mathbf{m}]}\end{array}$ & $\begin{array}{r}\text { Height } \\
{[\mathbf{m}]}\end{array}$ & $\begin{array}{r}\text { Impeller } \\
\text { diameter }[\mathbf{m}]\end{array}$ \\
\hline 1 & Mixer & 0.23 & 0.23 & 0.23 & 0.15 \\
1 & Settler & 0.23 & 0.90 & 0.23 & N.A. \\
2 & Mixer & 0.20 & 0.20 & 0.20 & 0.13 \\
2 & Settler & 0.20 & 0.80 & 0.20 & N.A. \\
\hline
\end{tabular}


Table 8: Optimal design of liquid-liquid extraction using mixer-settlers. Steps: $1=$ main extraction, $2=$ scrubbing.

\begin{tabular}{lrr}
\hline Variable & Step 1 & Step 2 \\
\hline No. stages & 4 & 3 \\
Aq. to org. flow ratio & 0.78 & 0.42 \\
[HNO $\left.{ }_{3}\right]$ aqueous scrubbing & N.A. & 5.2 \\
stream [M] & & \\
Impeller speed [rpm] & 200 & 200 \\
\hline
\end{tabular}

Table 9: Optimal design of liquid-liquid extraction using perforated plate pulsed column. Steps: $1=$ main extraction, $2=$ scrubbing. Plate spacing $=0.05 \mathrm{~m}$, plate free area $=23 \%$, hole diameter $0.003 \mathrm{~m}$. Minimum flooding: $70 \%$.

\begin{tabular}{lrr}
\hline Variable & Step 1 & Step 2 \\
\hline Height [m] & 0.47 & 3.17 \\
Diameter [m] & 0.23 & 0.20 \\
No. plates & 9 & 61 \\
Aq. to org. flow ratio & 0.41 & 0.22 \\
[HNO 3 ] aqueous scrubbing & N.A. & 4.5 \\
stream [M] & & \\
Pulse frequency [ $\mathbf{s}^{-1}$ ] & 0.67 & 0.55 \\
Pulse amplitude [m] & $1.77 \times 10^{-2}$ & $0.89 \times 10^{-2}$ \\
\hline
\end{tabular}




\section{Conclusions}

A case study in spent nuclear fuel reprocessing has been presented to com-

480 mixer-settlers and pulsed columns. To investigate the behaviour of the smallscale technology, models developed in a previous work have been used [26]. Mathematical models developed in the literature for specific aspects of the process (mass balance, thermodynamics, hydrodynamics, mass transfer coefficents, while only $40 \%$ is removed with pulsed columns. 
Small channels are safe in terms of criticality due to their geometry, resulting in small volume and a high surface area to volume ratio. Hence, their employment in the highly active section of the process, as an alternative to pulsed columns, should be considered more extensively. Small channels can lead to smaller volume required, easier control of $\mathrm{Np}$ and exhibit more efficient mass transfer.

\section{Acknowledgements}

The authors would like to acknowledge the UK Engineering and Physical Sciences Research Council (EPSRC) for funding provided as the PACIFIC project, EP/L018616/1. Davide Bascone would also like to thank University College London for his studentship.

\section{Nomenclature}

$\begin{array}{lll}\text { Symbols } & \text { Description } & \text { Units } \\ a & \text { Interfacial area } & \mathrm{m}^{2} \mathrm{~m}^{-3} \\ C & \text { Concentration } & \mathrm{mol} \mathrm{m}^{-3} \\ \text { ACapEx } & \text { annualised capital expenditure } & \mathrm{y}-1 \\ d_{32} & \text { Sauter diameter } & \mathrm{m} \\ d_{p} & \text { Drop diameter } & \mathrm{m} \\ D F & \text { Decontamination factor } & \mathrm{m} \\ \mathscr{D} & \text { Diffusion coefficient } & \mathrm{m}^{2} \mathrm{~s}^{-1} \\ \Delta H & \text { Plate spacing } & \mathrm{m} \\ E & \text { Extraction (unless differently stated) } & \\ L & \text { Length } & \mathrm{m} \\ k_{e f f} & \text { Effective multiplication factor } & \mathrm{m} \mathrm{s}^{-1} \\ k_{L} & \text { Mass transfer coefficient } & \\ m & \text { Distribution coefficient } & \\ N & \text { number of stage } & \\ N_{o c} & \text { number of transfer units } & \mathrm{y}^{-1} \\ O p E x & \text { Operating expenditure } & \end{array}$




$\begin{array}{lll}P e & \text { Peclet number } & \\ & \text { Reaction rate } & \mathrm{mol} \mathrm{L}^{-1} \mathrm{~s}^{-1} \\ S h & \text { Sherwood number } & \\ T & \text { Temperature } & \mathrm{K} \\ t & \text { time } & \mathrm{s} \\ T A C & \text { Total annaulised cost } & £ \mathrm{y}^{-1} \\ v & \text { Superficial velocity } & \mathrm{m} \mathrm{s}^{-1} \\ v_{t} & \text { Drop velocity for rising drop or falling drop } & \mathrm{m} \mathrm{s}^{-1} \\ V & \text { Volume } & \mathrm{m}^{3} \\ \dot{V} & \text { Volume flowrate } & \mathrm{m}^{3} \mathrm{~s}^{-1}\end{array}$

Greek Symbols

$\begin{array}{ll}\varphi & \text { Phase holdup } \\ \chi & \text { Recycle ratio of aqueous stream leaving step } \\ & 2\end{array}$

\section{Subscripts and superscripts}

$\begin{array}{ll}a q & \text { Aqueous phase } \\ e q & \text { Equilibrium } \\ k & \text { Generic phase } \\ i & \text { Generic component } \\ \text { in } & \text { Inlet } \\ m i x & \text { Mixer } \\ n & \text { Generic } \mathrm{n}^{\text {th }} \text { stages } \\ N & \text { Final } \mathrm{N}^{\text {th }} \text { stage } \\ \text { set } & \text { Settler }\end{array}$

Acronysms

HLW High Level Waste

PUREX Plutonium Uranium Extraction

SNF Spent Nuclear Fuel

TBP Tributyl Phosphate 


\section{References}

520

[1] T. A. Todd, Spent nuclear fuel reprocessing, in: Proc. of Nuclear Regulatory Commission Seminar, 2008 (2008).

[2] IAEA, Spent fuel reprocessing options, in: IAEA-TECDOC-1587, 2008 (2008).

${ }_{525}$ [3] R. T. Jubin, Spent fuel reprocessing, Tech. rep., Oak Ridge National Laboratory (2009).

[4] L. Borges Silverio, W. d. Q. Lamas, An analysis of development and research on spent nuclear fuel reprocessing, Energy Policy 39 (1) (2011) 281289 (Jan. 2011).

${ }_{530}^{5}$ [5] R. S. Herbst, P. Baron, M. Nilsson, Standard and advanced separation: PUREX processes for nuclear fuel reprocessing, in: K. L. Nash, G. J. Lumetta (Eds.), Advanced Separation Techniques for Nuclear Fuel Reprocessing and Radioactive Waste Treatment, Woodhead Publishing Series in Energy, Woodhead Publishing, 2011, pp. 141-175 (2011).

535

[6] G. L. Richardson, J. L. Swanson, Plutonium partitioning in the purex process with hydrazine stabilized hydroxylamine nitrate, Tech. rep., Hanford Engineering Development Laboratory (1975).

[7] J. D. Law, T. A. Todd, Liquid-liquid extraction equipment, Tech. Rep. INL/CON-08-15151, Idaho National Laboratory, United States (2008).

540 
[9] N. Assmann, A. Ładosz, P. R. v. Rohr, Continuous Micro Liquid-Liquid Extraction, Chemical Engineering \& Technology 36 (6) (2013) 921-936 (2013).

[10] K. Wang, G. Luo, Microflow extraction: A review of recent development, Chemical Engineering Science 169 (2017) 18-33 (Sep. 2017).

[11] D. Tsaoulidis, V. Dore, P. Angeli, N. V. Plechkova, K. R. Seddon, Extraction of dioxouranium(VI) in small channels using ionic liquids, Chemical Engineering Research and Design 91 (4) (2013) 681-687 (Apr. 2013).

[12] D. Tsaoulidis, V. Dore, P. Angeli, N. V. Plechkova, K. R. Seddon, Dioxouranium(VI) extraction in microchannels using ionic liquids, Chemical Engineering Journal 227 (2013) 151-157 (Jul. 2013).

[13] D. Tsaoulidis, P. Angeli, Effect of channel size on mass transfer during liquid-liquid plug flow in small scale extractors, Chemical Engineering Journal 262 (2015) 785-793 (Feb. 2015).

[14] P. Angeli, A. Gavriilidis, Hydrodynamics of taylor flow in small channels: A review, Proceedings of the Institution of Mechanical Engineers, Part C: Journal of Mechanical Engineering Science 222 (5) 737-751.

[15] F. P. Bretherton, The motion of long bubbles in tubes, Journal of Fluid Mechanics 10 (2) (1961) 166-188 (1961).

[16] W. Bier, W. Keller, G. Linder, D. Seidel, K. Schubert, H. Martin, Gas to gas heat transfer in micro heat exchangers, Chemical Engineering and Processing: Process Intensification 32 (1) (1993) 33-43 (Feb. 1993).

[17] J. M. Commenge, L. Falk, J. P. Corriou, M. Matlosz, Optimal design for flow uniformity in microchannel reactors, AIChE Journal 48 (2) (2002) 345-358 (Feb. 2002).

570

[18] C. Amador, A. Gavriilidis, P. Angeli, Flow distribution in different microreactor scale-out geometries and the effect of manufacturing tolerances and 
channel blockage, Chemical Engineering Journal 101 (1) (2004) 379-390 (2004).

[19] L. Luo, D. Tondeur, H. Le Gall, S. Corbel, Constructal approach and multiscale components, Applied Thermal Engineering 27 (10) (2007) 1708-1714 (Jul. 2007).

[20] M. Saber, J. M. Commenge, L. Falk, Rapid design of channel multi-scale networks with minimum flow maldistribution, Chemical Engineering and Processing: Process Intensification 48 (3) (2009) 723-733 (2009).

[21] J. M. Commenge, M. Saber, L. Falk, Methodology for multi-scale design of isothermal laminar flow networks, Chemical Engineering Journal 173 (2) (2011) 541-551 (2011).

[22] V. S. Koltunov, Kinetika reaktsii aktinoidov (Kinetics of Actinide Reactions), Atomizdat, Moscow, 1974 (1974).

[23] C. Hongyan, R. J. Taylor, M. Jobson, A. J. Masters, Simulation of neptunium extraction in an advanced purex process-model improvement, Solvent Extraction and Ion Exchange 35 (1) (2017) 1-18 (2017).

[24] J. E. Birkett, M. J. Carrott, O. D. Fox, C. J. Jones, C. J. Maher, C. V. Robeu, R. J. Taylor, D. A. Woodhead, Controlling neptunium and plutonium within single cycle solvent extraction flowsheets for advanced fuel cycles, Journal of Nuclear Science and Technology 44 (3) (2007) 337-343 (2007).

[25] S. Tachimori, Extra-m: A computing code system for analysis of the purex process with mixer settlers for reprocessing, Tech. rep., Japan Atomic Energy Research Institute (1994).

[26] D. Bascone, P. Angeli, E. S. Fraga, Mathematical modelling of intensified extraction for spent nuclear fuel reprocessing, Nuclear Engineering and Design 332 (2018) 162-172 (Jun. 2018). 
[27] R. Natarajan, N. K. Pandey, V. Vijayakumar, R. V. Subbarao, Modeling and Simulation of Extraction Flowsheet for FBR Fuel Reprocessing, Procedia Chemistry 7 (2012) 302-308 (2012).

[28] T. Asakura, S. Hotoku, Y. Ban, M. Matsumura, Y. Morita, Technetium separation for future reprocessing, Journal of Nuclear and Radiochemical Sciences 6 (3) (2005) 271-274 (Dec. 2005).

[29] M. Benedict, T. H. Pigford, H. W. Levi, Nuclear Chemical Engineering 2nd Edition, McGraw Hill, New York, 1980 (1980).

[30] S. Kumar, S. B. Koganti, Modelling of np(iv) np(vi) distribution coefficients in $30 \% \mathrm{tbp} / \mathrm{n}$-dodecane/nitric acid/water biphasic purex system, Indian Journal of Chemical Technology 8 (1) (2001) 41-43 (Jan. 2001).

[31] G. Uchiyama, S. Hotoku, S. Fujine, Distribution of nitrous acid between tri-n-butyl phosphate/ n-dodecane and nitric acid, Solvent Extraction and Ion Exchange 16 (5) (1998) 1177-1190 (1998).

[32] R. D. Carter, G. R. Kiel, K. R. Ridgway, Criticality handbook vol. 1, Tech. rep., Atlantic Richfield Hanford Co. Report ARH-600 (1968).

[33] M. N. Kashid, A. Gupta, A. Renken, L. Kiwi-Minsker, Numbering-up and mass transfer studies of liquid-liquid two-phase microstructured reactors, Chemical Engineering Journal 158 (2) (2010) 233-240 (Apr. 2010).

[34] M. N. Kashid, D. W. Agar, Hydrodynamics of liquid-liquid slug flow capillary microreactor: flow regimes, slug size and pressure drop, Chemical Engineering Journal Vol. 131, 1-13 (2007).

[35] CM Scientific, http://www.cmscientific.com/, accessed on 23/10/2017.

[36] A. Tonkovich, D. Kuhlmann, A. Rogers, J. McDaniel, S. Fitzgerald, R. Arora, T. Yuschak, Microchannel technology scale-up to commercial capacity, Chemical Engineering Research and Design 83 (A6) (2005) 634639 (2005). 
[37] F. Scheiff, M. Mendorf, D. Agar, N. Reis, M. Mackley, The separation of immiscible liquid slugs within plastic microchannels using a metallic hydrophilic sidestream, Lab Chip 11 (6) (2011) 1022-1029 (2011).

[38] K. Gonda, S. Miyachi, S. Fukuda, Mass Transfer Model of Purex Process in Mixer-Settlers, Journal of Nuclear Science and Technology 23 (5) (1986) 472-474 (1986).

[39] B. Hanson, Process engineering and design for spent nuclear fuel reprocessing and recycling plants, in: R. Taylor (Ed.), Reprocessing and Recycling of Spent Nuclear Fuel, Woodhead Publishing Series in Energy, Woodhead Publishing, Oxford, 2015, pp. 125 - 151 (2015).

[40] R. Treybal, Liquid Extraction, McGraw Hill Book Company Inc., New York, 1963 (1963).

[41] F. S. Gharehbagh, S. M. A. Mousavian, Hydrodynamic characterization of mixer-settlers, Journal of the Taiwan Institute of Chemical Engineers 40 (3) (2009) 302-312 (May 2009).

[42] P. G. Alfredson, B. G. Charlton, R. K. Ryan, V. K. Vilkaitis, Development of processes for pilot plant production of purfied uranyl nitrate solutions, Tech. rep., Australian Atomic Energy Commission (1975).

[43] W. D. Seider, J. D. Seader, D. R. Lewin, Product and process design principles: synthesis, analysis, and evaluation, Wiley, New York, 2004 (2004).

[44] L. Steiner, Computational procedure for column simulation and design, in: J. C. Godfrey, M. J. Slater (Eds.), Liquid-Liquid Equipment, John Wiley \& Sons Ltd, 1994, pp. 116-135 (1994).

[45] K. Gonda, T. Matsuda, Solvent Extraction Calculation Model for Purex Process in Pulsed Sieve Plate Column, Journal of Nuclear Science and Technology 23 (10) (1986) 883-895 (1986). 
[46] A. Kumar, S. Hartland, Computational Strategies for Sizing Liquid-Liquid Extractors, Industrial \& Engineering Chemistry Research 38 (3) (1999) 1040-1056 (Mar. 1999).

[47] T. Matsuda, K. Gonda, Motion of Dispersed Drops and Mass Transfer in Pulsed Column, Journal of Nuclear Science and Technology 23 (7) (1986) 633-642 (1986).

[48] A. Kumar, S. Hartland, Empirical prediction of operating variables, in: J. C. Godfrey, M. J. Slater (Eds.), Liquid-Liquid Equipment, John Wiley \& Sons Ltd, 1994, pp. 625-735 (1994).

[49] A. C. Jealous, H. F. Johnson, Power Requirements for Pulse Generation in Pulse Columns, Industrial \& Engineering Chemistry 47 (6) (1955) 11591166 (Jun. 1955).

[50] R. Smith, Chemical Process Design and Integration, John Wiley \& Sons Ltd, United Kingdom, 2005 (2005).

[51] OECD/NEA, Nuclear energy data (2007).

[52] GAMS Development Corporation, General Algebraic Modeling System (GAMS) Release 24.2.1, Washington, DC, USA (2013).

[53] V. Dore, D. Tsaoulidis, P. Angeli, Mixing patterns in water plugs during water/ionic liquid segmented flow in microchannels, Chemical Engineering Science 80 (2012) 334-341 (Oct. 2012).

[54] D. Tsaoulidis, P. Angeli, Effect of channel size on liquid-liquid plug flow in small channels, AIChE Journal 62 (1) (2016) 315-324 (Jan. 2016).

[55] B. Guillaume, J. P. Moulin, C. Maurice, Chemical properties of neptunium applied to neptunium management in extraction cycles of purex process, in: Extraction '84, Pergamon, 1984, pp. 31-45 (1984). 
[56] E. G. Ortega, D. Tsaoulidis, P. Angeli, Predictive model for the scale-out of small channel two-phase flow contactors, Chemical Engineering Journal 351 (2018) $589-602$ (2018). 